\title{
Metal Free Dual Modal Contrast Agents based on Fluorographene Quantum Dots
}

Sruthi Radhakrishnan ${ }^{1}$, Atanu Samanta ${ }^{2}$, Parambath M. Sudeep ${ }^{1}$, Kiersten L. Maldonado ${ }^{3}$, Sendurai A. Mani ${ }^{4}$, Ghanashyam S. Acharya ${ }^{5}$, Chandrasekhar S. Tiwary ${ }^{1, *}$, Abhishek K. Singh ${ }^{2, *}$, Pulickel M. Ajayan ${ }^{1, *}$

${ }^{1}$ Department of Materials Science and NanoEngineering, Rice University, Houston, Texas, USA-77005

${ }^{2}$ Materials Research Center, Indian Institute of Science, Bangalore, India-560012.

${ }^{3}$ Small Animal Imaging Facility, The University of Texas MD Anderson cancer center, Houston, Texas.

${ }^{4}$ Department of Translational molecular pathology, Division of pathology/lab medicine, The University of Texas MD Anderson cancer center, Houston, Texas.

${ }^{5}$ Department of Ophthalmology, Baylor College of Medicine, Houston, Texas.

*Address correspondence to: ajayan@ rice.edu (P.M.A.), abhishek@mrc.iisc.ernet.in (A.K.S.), cst311@gmail.com(C.S.T.)

Keywords: Fluorinated graphene Quantum dots, Magnetic Resonance Imaging(MRI), Graphene, DFT Calculations, Contrast agent

Fluorographene quantum dots prepared from fluorinated graphene oxide (FGO) show a linear dependence of the magnetization on the applied field. This is further supported by DFT calculations taking into account a few possible systems of functionalized graphene quantum dots. The inherent magnetism, high concentration of fluorine and cyto-compatibility of these quantum dots promise potential application as a dual modal agent for proton and 19F based Magnetic Resonance Imaging which is investigated here. A metal free dual modal contrast agent would bring about a great change in the efficiency and resolution of this widely used imaging tool.

\section{Introduction}

Carbon and its allotropes have been studied for their unique properties, which offer immense possibilities for future technological applications in energy devices, solar cells and medical applications [1-3]. Magnetism in carbonaceous materials are of immense importance due their unprecedented applications in spintronics, and bio-sensors [4,5]. Graphene, a member of the carbon family, in the absence of any d or f orbital electrons is inherently diamagnetic [6].Recent 
reports show that the possibility of the magnetism in graphene can be introduced by defects, adatoms or edge states [7-13]. The magnetic ordering due to defects has been attributed to the mixture of $\mathrm{sp}^{2}-\mathrm{sp}^{3}$ bonding [7-11]. Spin half states at the edges of zigzag graphene [12] or a negative curvature in graphitic plane consisting of a few rings can introduce magnetic ordering [13]. Adatoms in graphene, like fluorine carry unpaired moments resulting in paramagnetic behavior [7].

Graphene confined to quantum dimension form stable, luminescent colloidal suspension known as graphene quantum dots (GQDs). Many theoretical predictions and calculations have been made towards the magnetism in graphene quantum dots $[6,12,14-15]$. Bhowmick et. al. showed the robustness of edge state magnetism in graphene nanostructures [12]. In a recent work by Sun et. al., a weak paramagnetism is reported in graphene oxide quantum dots [16]. It has been observed that the edge states as well as the hydroxyl groups contribute to the paramagnetism. This paramagnetism in graphene due to edges and functional groups could be further enhanced by fluorine substitution in the lattice as reported by R.R Nair et.al [7].In a recent work from our group, fluorinated graphene oxide (FGO) a novel material derived from fluorinated graphite polymer (FG) was reported to be paramagnetic [17]. This received a lot of attention as it was the first reported "magnetic nanoparticle free carbon based contrast agent for Magnetic Resonance Imaging (MRI)" [18].

MRI is a powerful and commonly used imaging tool, due to its high spatial resolution and nonionizing radiations [19]. Gadolinium $\left(\mathrm{Gd}^{3+}\right)$ based contrast agents lead the market of MRI contrast agents in spite of their high inherent toxicity [20,21]. These agents are rapidly removed by renal clearance. As a result, the imaging window available with these agents is very short. This makes repeated administration ineludible for multiple imaging sessions [22]. The poor stability of 
Gadolinium chelates release Gadolinium ions which accumulate in the brain causing long term functional and toxicological effects. Reports suggests that Gadolinium based agents can induce Nephrogenic Systemic Fibrosis(NSF) in patients with renal or vascular disorders [23]. Several studies in vitro, on human skin fibroblasts and proximal tubular epithelial cells also demonstrate the high toxicity of Gadolinium based agents [24,25]. Also these agents modify the contrast in an image. Therefore, for unambiguous detection of the localization of contrast agent in vivo, it is essential to have an image before and after injection of the agent. This is extremely difficult and laborious [26].

Other contrast agents based on Iron and Manganese has also garnered interest recently [22]. In Manganese based agents it is extremely difficult to achieve chelation stability. Some Manganese based contrast agents have shown detectable toxicity within two weeks of administration. Owing to these limitations only one manganese based agent, commercially available as Teslascan is approved for clinical use [23]. Iron oxide based agents confer negative contrast making it indispensable to have pre and post injection images for direct evaluation of the images [26].

These limitations call for an urgent and unmet need for a metal free contrast agent with less toxicity. FGO being metal free, is supposed to be less toxic for biological systems. However, the large size of these sheets limits its translation to in-vivo use as these will cut or block the capillaries proving to be fatal. Thus it is vital to study the magnetic behavior of fluoro graphene quantum dots having nanometric dimensions. Most importantly, these quantum dots could be developed as a dual modal agent for MRI and 19F MRI. This would avoid the multiple imaging required while using $\mathrm{Gd}^{3+}$ based agents without compromising on efficiency.

There have been reports for dual modal agents for ultrasound and 19F MRI [27], but this is presumably the first report of a metal free nanomaterial that could be used as a dual modal agent 
for proton and 19F MRI. The efforts in the development of a dual modal agent for ${ }^{19} \mathrm{~F}$ and proton MRI has been focused on introducing $\mathrm{Gd}^{3+}$ ion into fluorinated materials. However, this relaxes the Fluorine faster making it unable to be detected by 19F MRI [28]. To surmount these limitations, there is an urge to study the magnetic property of Fluoro graphene quantum dots. It could open up the doors for a metal free, cyto-compatible, dual modal agent with higher spatial resolution.

In this work we explore the magnetic behavior of fluoro graphene quantum dots prepared from FGO. The experimental observations are well supported by theoretical calculations. The structural and magnetic characterization of these quantum dots were carried out. The low temperature magnetic measurements do not show any signature of magnetic ordering. The temperature dependent susceptibility of FGO derived quantum dots follow Curie's law, which confirms their paramagnetic nature. DFT studies show that this paramagnetism arises due to fluorine adatoms and presence of functional groups. Each fluorine adatom and functional groups carry $1 \mu_{\mathrm{B}}(\mathrm{Bohr}$ Magneton). This magnetic moment induces from the quasilocalized state, which is arising from the absorbed functional groups. The MRI relaxivity was measured to assess the relaxation rate and feasibility of these quantum dots as a contrast agent. The biocompatibility studies on human derived cell lines were carried out by MTS assay.

\section{Results and Discussion}

Fluoro graphene quantum dots (FGQd) were prepared from FGO by simple liquid exfoliation [29]. FGO was prepared by the oxidation of FG by an improved method [30]. The synthesis of FGO yields two products, a hydrophilic less fluorinated graphene oxide which settles to the bottom and a hydrophobic highly fluorinated graphene oxide which separates to the top. The highly fluorinated part, which separates to the top was then washed and used to prepare the quantum dots. The quantum dots were synthesized by a top down approach [29]. FGO was bath sonicated in N-N 
Dimethyl formamide (DMF) for 6 hours. The hydrodynamic forces help to break down the larger flakes into smaller ones [31]. The top three quarter of the solution, which would contain the smaller flakes was decanted. This was stirred on a hot plate at $110^{\circ} \mathrm{C}$ for 6 hours. A disperse solution of the quantum dots and flakes in DMF was obtained. The quantum dots were separated by centrifugation at 10,000rpm for 30minutes. A schematic illustration of this synthesis is shown in figure 1 .

The structural characterization of the quantum dots was carried out using Transmission Electron Microscope (TEM). The size varied from 8-12 nm while most of the dots were of the size $10 \mathrm{~nm}$. The size varied from 8-12 $\mathrm{nm}$ while most of the dots were of the size $10 \mathrm{~nm}$. The size measured by Dynamic Light Scattering (DLS) is shown in Figure S1 of the Supporting Information. DLS however indicates the size of the aggregates rather than individual particles. In the HRTEM shown in figure 2, a good superlattice structure with discoidal in shape can be seen. From the HRTEM, it can be inferred that FGQds are single layered as a consequence of the rigorous exfoliation during the oxidation process combined with the exfoliation to quantum dots. The single layered structure obviates the possibility of spin pairing of fluorine in the adjacent layers.

Raman spectroscopy was used to get more structural information about the quantum dots. It shows many characteristics corresponding to high defect density graphene. The defects or the edges in graphene are capable of giving the D peak [32-35]. In the Raman spectra given in figure $2 b$ the high D peak with an Id/IG ratio of 1.093 is arising from the increasing defects in the lattice due to the Fluorine doping and due to the higher number of edges in the quantum dots [36-38]. On comparison of the $\mathrm{I}_{\mathrm{D}} / \mathrm{I}_{\mathrm{G}}$ ratio to that of graphene quantum dots $[39,40]$ fluro-graphene quantum dots have higher number of defects than graphene quantum dots. These are contributed by the $\mathrm{sp}^{3}$ $\mathrm{C}$ atoms bonded to the fluorine. The $\mathrm{G}$ band seems to be upshifted from $1580 \mathrm{~cm}^{-1}$ to $1600 \mathrm{~cm}^{-1}$. At 
higher defect densities, the $\mathrm{G}$ and the $\mathrm{D}$ ' band becomes too wide to form a single upshifted $\mathrm{G}$ band which is seen in this case [36]. The appearance of 2D peak which is a doublet peak of the D band and the $\mathrm{D}+\mathrm{D}^{\prime}$ band too is due to the high defects in the Quantum dots.

However, Raman spectroscopy does not give any information about the functional groups. Since the quantum dots are derived from the functionalized derivative FGO, there could be functional groups present. The presence of functional groups was verified from the IR spectrum (Figure 2c). The spectrum shows a characteristic $\mathrm{C}=\mathrm{O}$ peak and a broad $-\mathrm{OH}$ peak showing the presence of acid functional groups. The entire spectrum shown in the inset indicates the presence of C-F bond. The optical property of the quantum dots studied using UV-Vis spectroscopy (Figure S2, supporting Information) shows the peak corresponding to $\pi-\pi^{*}$ transition of C-C bond at $225 \mathrm{~nm}$. FG2 shows an additional peak corresponding to the $n-\pi^{*}$ transition of carbonyl groups which confirms the presence of functional groups.

A qualitative analysis was carried out by X-ray Photoelectron Spectroscopy (XPS). The deconvoluted XPS spectrum is shown in Figure 3a. C1s spectrum peak at $284.1 \mathrm{eV}$ corresponds to the $\mathrm{sp}^{2}$ carbon in graphene; $285.83 \mathrm{eV}$ agrees closely with that of ionic C-F bond. The F1s shows peak at $683.8 \mathrm{eV}$ which confirms the ionic nature of the bond with Carbon [37,38,41,42]. XPS analysis do not show the presence of any magnetic impurities like Iron. The XPS data shows that the relative fluorine content is $4.87 \%$. It is reported that, at such low concentrations of fluorine, the carbon fluorine bond tends to be ionic, which is seen in this case [43].

A detailed magnetic characterization is performed to explore the magnetism in these fluorine doped quantum dots (figure $3 \mathrm{c})$. The calculated susceptibility $(\chi=\mathrm{M} / \mathrm{H})$ with temperature $(\mathrm{T})$ follows Curie's law $(\chi \propto 1 / \mathrm{T})$ as shown in figure $3 \mathrm{~d}$, which indicates fluorinated graphene quantum dots are purely paramagnetic nature. At low temperature, the inverse of susceptibility varies 
linearly with temperature (Figure $3 \mathrm{~d}$ inset), which also support that the samples have purely paramagnetic nature with no magnetic ordering. The $\mathrm{M}-\mathrm{H}$ curve was measured at room temperature and the diamagnetic contribution of the background has been subtracted from measured magnetization (Figure 3c). FG shows a linear dependence of magnetization on the applied magnetic field. To further understand the magnetic properties of functionalized graphene quantum dots, we have modeled the real system by considering a $3 \times 3 \times 1$ supercell of graphene in the first-principle density functional theory (DFT) calculations $[44,45]$. The details of the calculation are described in the theoretical method section. According to Lieb's second theorem, if the total number of electrons of a bipartite system is odd then the total magnetic moment of the system in the ground states has to be $M s=\frac{\mu B(N \alpha-N \beta)}{2}$ (where, $\left(\mathrm{N}_{\alpha}-\mathrm{N}_{\beta}\right)$ is the difference between the number of atoms in each sublattice), which is independent of dimensionality and periodic lattice structure. This theorem indicates that the ground state magnetic properties of graphene quantum dots will not be much difference from the periodic graphene lattice. The calculated total energy difference between magnetic and non-magnetic systems is shown in figure $4 \mathrm{a}$. The magnetic solution of each functionalized graphene is lower in energy compared to the nonmagnetic state, which indicates that this functional group will introduce magnetism in the graphene. Each functional group contributes nearly $1 \mu_{\mathrm{B}}$ (Figure $4 \mathrm{~b}$ ), which is in well agreement with theoretical prediction [44]. To further understand the magnetism of functionalized graphene, spin charge density of functionalized graphene was calculated (Figure 4c-4e). The spin up charge density is localized around the functionalization group, which indicates that this magnetic center will behave like a paramagnet. This kind of charge localization is also observed in defected $\mathrm{H}$ and $\mathrm{F}$ functionalized graphene, which act as a paramagnetic center [7,46-49]. Moreover, the calculated bond length of $\mathrm{C}-\mathrm{F}$ and $\mathrm{C}-\mathrm{O}$ in $\mathrm{F}$ and $\mathrm{OH}$ functionalized graphene are $1.55 \AA$ and $2.80 \AA$, 
respectively. This indicates that $\mathrm{F}$ and $\mathrm{OH}$ functional group are covalently bonded with graphene carbon atom resulting in an $\mathrm{sp}^{2}-\mathrm{sp}^{3}$ bonded atomic network, which is seen in the XPS analysis. The distance between $\mathrm{C}, \mathrm{O}$ and $\mathrm{H}$ of $\mathrm{COOH}$ functional group and graphene carbon atom are 3.69 $\AA$, 3.30-3.58 $\AA$ and $2.59 \AA$, respectively, which indicates that the $\mathrm{COOH}$ functional group is chemically adsorbed into graphene. Furthermore, the magnetic solution of $\mathrm{OH}$ and $\mathrm{F}$ functionalized graphene shows lower in energy when $\mathrm{OH}$ and $\mathrm{F}$ functional groups are adsorbed on the same sublattice of graphene. However, if $\mathrm{OH}$ and $\mathrm{F}$ functional groups are present in different sublattices of graphene, then non-magnetic solution is favored as shown in figure $4 \mathrm{~b}$. This finding is in good agreement with Leib's theorem [46]. The presence of both functional group slightly decrease the magnetic moment of the system as shown figure $4 \mathrm{~b}$. This suggest that the magnetic system of graphene is strongly depends on the concentration, presence of functional groups and absorption sites. Moreover, when $\mathrm{OH}$ and $\mathrm{F}$ functional groups are attached to neighboring carbon atoms of graphene, an unstable $\mathrm{FOH}$ molecule is formed, which results in a non-magnetic state. A stable $\mathrm{CO}_{2}$ and $\mathrm{HF}$ molecule formation is found when $\mathrm{COOH}$ and $\mathrm{F}$ functional group is present in the graphene. This suggests that $\mathrm{COOH}$ functional groups have less chance to be on the surfaces of the graphene monolayer.

The magnetic behavior of a paramagnetic system is described by the Brillouin function as

$$
M=N g J \mu B\left[\frac{(2 J+1)}{2 J} \operatorname{coth}\left(\frac{(2 J+1) x}{2 J}\right)-\frac{1}{2 J} \operatorname{coth}\left(\frac{x}{2 J}\right)\right]----(1)
$$

Where $z=g J \mu b H k$ and $\mathrm{x}=\left(\mathrm{gJ} \mu_{\mathrm{B}} \mathrm{H} / \mathrm{k}_{\mathrm{B}} \mathrm{T}\right)$.

$M, n, g, \mathrm{~J}, \mu_{\mathrm{B}}, H, T, \mathrm{k}_{\mathrm{B}}$ is the magnetization of the sample, number of spin(free), $g$-factor, theangular momentum, Bohr magneton, applied magnetic field, absolute temperature and Boltzmann constant, respectively. Since the DFT calculation also shows that each functional group contributes $1 \mu_{\mathrm{B}}$, the experimental M Vs $\mathrm{H}$ data is fitted with Eq. (1) by considering $\mathrm{J}=\mathrm{S}=0.5$ (for 
free electron). The equation (1) is best fitted with $\mathrm{n}$ value $3.2 \times 10^{20} \mathrm{gm}^{-1}$, which is higher than the XPS measure the fluorine concentration $1.38 \times 10^{20} \mathrm{gm}^{-1}$. This discrepancy could be possible due to presence of other fictional groups and defects.

These magnetic centers in graphene, introduced by functional groups is observed in graphene oxide quantum dots [16]. However, the measured magnetization of the Graphene Oxide quantum dots starts saturating at 3T, while that of FGQds do not saturate upto the applied field of 5T. The paramagnetism along with this high value of saturation magnetism, could make these ideal agents for T2 contrast in MRI. In MRI imaging T2 measures the time required for the decay of the transverse magnetization induced by the RF pulse. The T2 relaxation was measured in a Bruker 4.7T, with 30 echo times from $10 \mathrm{~ms}$ to $300 \mathrm{~ms}$. Pure water has a T2 relaxation of $\sim 650 \mathrm{~ms}$. On the introduction of the quantum dots it reduced to $378 \mathrm{~ms}$. The plot showing the transverse magnetization decay is shown in supporting information Figure S3. The image acquired during MRI is shown in figure 5a. 19F MRI is based on the relaxation of F19 nuclei. Mobile fluorine is present in the body at very low concentrations which are below the detection limit. High concentration of fluorine is present in the bones which are immobilized and hence cannot be detected. Thus 19F MRI has the advantage of very low background signal and the combination of proton and 19F MRI eliminates the need for pre and post scans required for Gadolinium based agents [24]. 19F MRI scans were conducted on the proton MRI system by modulating the resonance frequency. Signals could be picked up and an image could be generated at concentrations as low as $10 \mu \mathrm{g} / \mathrm{mL}$ (Figure $5 \mathrm{~b}$ ).

Calculations show that the relaxation rate $\mathrm{r} 2$ for FGQds is $39 \mathrm{~s} / \mathrm{g} / \mathrm{mL}$. The proposed mechanism of interaction of the protons and the paramagnetic agents is illustrated graphically in Figure 5c. The paramagnetic agents interact with the applied magnetic field and act as centers of local magnetic 
field. This causes inhomogeneity in the magnetic field which interacts with the protons in water shortening their relaxation time. This value of relaxation is comparable to a commercial available, Gadolinium based contrast agent, Ablavar, previously marketed under the name MS 325 [50]. It is used for the evaluation of aortoiliac occlusive disease in adults with known or suspected peripheral vascular disease. An insight into the currently reported carbon based or graphene based contrast agents for MRI reveals that all of them are metal based agents. For instance, FeMn-GO with Iron and Manganese embedded in Graphene oxide [22] and Iron oxide-GO composites [51] have relaxivities in the order of $100 \mathrm{mM} / \mathrm{s}$. The toxicological issues associated with these metallic agents covers their high $\mathrm{r} 2$ values.

A preliminary assessment of cytocompatibility is an indispensable part of this study. The cytocompatibility (Figure. 5d) of the quantum dots were measured by MTS assay. For the study, HEK 293 T cells were incubated with the quantum dots for 1,2 and 3 days. At the end of the incubation time MTS reagent was added to the cells, and incubated for one more hour and the optical density was read with a micro plate reader at $490 \mathrm{~nm}$. The cells incubated in the growth media under identical environment was used as the control. The optical density reflects the number of viable cells. The results do not indicate any dose dependent toxicity. Even at the highest concentration no significant cell death is seen. Even after 3 days there is no considerable decrease in the viability, which rules out the possibility of incubation time dependent toxicity. The observation is further confirmed by cellular imaging GFP labelled SUM 159 cells were seeded at 1000cells/well and incubated with the quantum dots under identical conditions. A Zeiss axiovision A1 microscope with Axiovision software was used to capture the images after 1,2 and 3 days of incubation. Figure $5 e$ and $5 \mathrm{f}$ shows dark field fluorescent images of the cells after 1 day and 3 days 
of incubation respectively. The inset shows an overlapped image of the bright field and fluorescent image. The cells look viable and no proliferation defects are seen.

\section{Conclusion}

Magnetic Resonance Imaging (MRI) is a powerful imaging tool in the clinical biomedical imaging for disease diagnosis. It is based on the relaxation of protons in water due to the local magnetic field experienced by them. The "gold standard" contrast agents used today are Gadolinium or Iron based, which require post and pre images for efficient detection. Moreover, these metal based agents pose a threat of high toxicity with repeated administrations. The Fluoro graphene quantum dots synthesized from FGO possess novel attributes suitable for its use in imaging. The inherent paramagnetism with the presence of Fluorine enables its use as a dual modal agent for proton and 19F MRI. This avoids the pre and post scan sessions required with conventional agents. The presence of functional groups and small size will enhance the chances of internalization of these agents into the tissues giving higher retention times and imaging efficiency than the conventionally used metallic contrast agents.

\section{Experimental Section}

Synthesis: Fluorinated graphene oxide was prepared by dispersing the Fluorinated Graphite polymer (FG)in a 9:1 mixture of concentrated sulfuric acid and concentrated phosphoric acid. The mixture was stirred at $50^{\circ} \mathrm{C}$ for 2 hours followed by the addition of $6 \mathrm{gm}$ equivalent of Potassium permanganate. On addition of potassium permanganate, the temperature of the mixture rises to $90^{\circ} \mathrm{C}$. The mixture was left to stir overnight. The mixture was poured over ice and $10-12 \mathrm{ml}$ of hydrogen peroxide was added to it. On the addition of hydrogen peroxide, a light brown part settles on the bottom while a dark brown part separates to the top. The separation was allowed to complete by keeping the mixture undisturbed overnight. This observed phase separation is due to the 
difference in fluorine content. The top portion is highly fluorinated which makes it hydrophobic, while the bottom portion is less fluorinated and hydrophilic part.

Magnetic measurements: The sample was sealed in vacuum in NMR tubes and the hysteresis curve was measured using a SQUID magnetometer at room temperature. Liquid samples were used for the measurement at the as prepared concentration. The background with the solvent was measured independently to eliminate the possibility of ferromagnetic impurities and was found to be diamagnetic.

Cyto-compatibility: MTS assay was used to assess the cyto-compatibility of the quantum dot samples. These studies were conducted by serial dilution of the quantum dots. HEK 293T cells cultured in DMEM cell culture media substituted with FBS and penicillin and Streptomycin was used for the studies. 1000 cells were seeded per well in a 96 well plate and the quantum dots were added to these with serial dilution. This was incubated at $37^{\circ} \mathrm{C}$. After incubating it for 1 day, 2 days and 3days the absorbance values were measured after the addition of MTS reagent. The fluorescent images were obtained using GFP labelled SUM 159 cells incubated under identical conditions. MRI Relaxation time: The relaxation time measurements were conducted in a $4.7 \mathrm{~T}$ Biospec system. The measurement was conducted by immersing the solution containing the quantum dots, sealed in ultra-small centrifuge in water with Magnevist serving as a positive control.

Theoretical methodology: All the theoretical calculations were performed using first-principle density functional theory (DFT) as implemented in the VASP package [46]. The exchange and correlation effects of electrons is approximated by using generalized gradient approximation Perdew-Burke-Ernzerhof (PBE) functional [47,48]. The frozen core approximation using projector-augmented wave ( PAW ) pseudo-potentials is used to represent the electrons and ion interaction [47]. The electronic wave functions are represented by the plane wave basis set with 
$400 \mathrm{eV}$ energy cutoff. To model $\mathrm{F}, \mathrm{OH}, \mathrm{COOH}$ functionalized graphene system, a 3x3x1 graphene supercells having a $20 \AA$ vacuum along the $c$ axis is used in order to avoid any interaction between periodically repeated images. The reciprocal space was sampled by 5x5x1 Monkhorst-Pack $k$-point grids [50]. The final structures are relaxed unless the forces on each atom becomes less than 0.01 eV/ A. It has been observed that the DFT theory within semi-local GGA approximation PBE functional studies of $\mathrm{H}$ and $\mathrm{F}$ functionalized graphene gives rise to a nonmagnetic states [53,54]. This semi-local GGA functional reduces delocalized electronic states due to their spurious inherent self-interaction error (SIE), which results to a nonmagnetic state of the functionalized graphene. The hybrid functionals such as PBE0 and HSE has shown good predictability of magnetic properties of functionalized graphene [55]. Here, we have employed the PBE0 functional, where exchange and correlation is approximated as follows [55].

$$
E_{x C}^{P B E 0}=E_{x c}^{P B E}+\frac{1}{4}\left(E_{x}^{H F}-E_{x}^{P B E}\right)
$$

This Hybrid functional used 25\% of HF with $75 \%$ of PBE exchange, which is orbital dependent i.e. non-local in space and greatly reduces the spurious inherent self-interaction error. The PBE relaxed structures are considered for the PBE0 functional calculations.

\section{Supporting Information}

Supporting Information is available online from the Wiley Online Library or from the author.

\section{Acknowledgements}

This material is based upon work supported by a Hamill IBB Innovations Award Grant funded by the Hamill Foundation and the Air Force Office of Scientific Research award FA9550-12-1-0035.

Received: ((will be filled in by the editorial staff))

Revised: ((will be filled in by the editorial staff)) Published online: ((will be filled in by the editorial staff)) 
[1] Su, D. S.; Centi, G. A perspective on carbon materials for future energy applications. $J$. Energy chem. 2013, 22, 151-173.

[2] Krueger, A. New carbon materials biological applications of functionalized nanodiamond materials. Chem. Eur. J. 2008, 14, 1382-1390.

[3] Zhu, H.; Wei, J.; Wang, K.; Wu, D. Applications of carbon materials in photovoltaic solar cells. Sol. Energy Mater. Sol. Cells 2009, 93, 1462-1470.

[4] Han, W.; Kawakami, R. K.; Fabian, M. G. J. Graphene Spintronics. Nat. Nanotechnol. 2014, 9, 794-807.

[5] Bogani, L.; Wernsdorfer, W. Molecular spintronics using single-molecule magnets. Nat. Mater. 2008, 7, 179-186

[6] Fernandez-Rossier, J.; Palacios, J. J. Magnetism in Graphene Nanoislands. Phys. Rev. Lett. 2007, 99, 1-4.

[7] Nair, R. R.; Sepioni, M.; Tsai, I.-L.; Lehtinen, O.; Keinonen, J.; Krasheninnikov, A. V.; Thomson, T.; Geim, A. K.; Grigorieva, I. V. Spin-Half Paramagnetism in Graphene Induced by Point Defects. Nat. Phys. 2012, 8, 199-202.

[8] Červenka, J.; Katsnelson, M. I.; Flipse, C. F. J. Room-Temperature Ferromagnetism in Graphite Driven by Two-Dimensional Networks of Point Defects. Nat. Phys .2009, 5, 840-844.

[9] Chen, J.-H.; sCullen, W. G.; Williams, E. D.; Fuhrer, M. S. Tunable Kondo Effect in Graphene with Defects. Nat. Phys.2010, 7, 22.

[10] Sepioni, M.; Nair, R. R.; Rablen, S.; Narayanan, J.; Tuna, F.; Winpenny, R.; Geim, A. K.; Grigorieva, I. V. Limits on Intrinsic Magnetism in Graphene., Phys. Rev. Lett., 2010, $207205,1-4$. 
[11] Wang, W. L.; Meng, S.; Kaxiras, E. Graphene NanoFlakes with Large Spin. Nano Lett. 2008, 8, 244-245.

[12] Bhowmick, S.; Shenoy, V. B. Edge State Magnetism of Single Layer Graphene Nanostructures. J. Chem. Phys. 2008, 128.

[13] Park, N.; Yoon, M.; Berber, S.; Ihm, J.; Osawa, E.; Tománek, D. Magnetism in AllCarbon Nanostructures with Negative Gaussian Curvature. Phys. Rev. Lett. 2003, 91, 237204.

[14] Potasz, P.; Güçlü, A. D.; Wójs, A.; Hawrylak, P. Electronic Properties of Gated Triangular Graphene Quantum Dots: Magnetism, Correlations and Geometrical Effects. Phys. Rev. B - Condens. Matter Mater. Phys. 2012, 85, 1-11.

[15] Wang, W. L.; Meng, S.; Kaxiras, E. Graphene Nanoflakes with large spins. Nano Lett. 2008, 8, 241-245.

[16] Sun, Y.; Zheng, Y.; Chen, J.; Zhang, W.; Tang, N.; Du, Y. Intrinsic Magnetism of Monolayer Graphene Oxide Quantum Dots. Appl. Phys. Lett. 2016, 108, 033105.

[17] Romero-Aburto, R.; Narayanan, T. N.; Nagaoka, Y.; Hasumura, T.; Mitcham, T. M.; Fukuda, T.; et al. Fluorinated Graphene Oxide: A New Multimodal Material for Biological Applications. Adv. Mater. 2013, 25, 5632-5637.

[18] $\mathrm{Hu}, \mathrm{Y}$. H.; The first magnetic-nanoparticle-free Carbon based contrast agent of Magnetic Resonsce Imaging-Fluorinated Graphene Oxide. Small, 2012.

[19] Guzman, R.; Uchida, N.; Bliss T. M.; He, D. P.;Christopherson, K.K.; et al. Long-term monitoring of transplanted human neural stem cells in developmental and pathological contexts with MRI. Proc Natl Acad Sci. 2007, 104, 10211-10216.

[20] Ersoy, H.; Rybicki, F. J.Biochemical safety profiles of gadolinium-based extracellular 
contrast agents and nephrogenic systemic fibrosis. J. Magn. Reson. Imaging. 2007, 26, 1190-1197.

[21] Zhitong, Z.; Lin, M.; Hengjin, L. Incidence of nephrogenic systemic fibrosis at Chinese PLA General Hospital. J. Magn. Reson. Imaging 2009, 30, 1309-1312.

[22] Kanakia, S. et al. Towards An Advanced Graphene-Based Magnetic Resonance Imaging Contrast Agent: Sub-acute Toxicity and Efficacy Studies in Small Animals. Sci. Rep. 2015, 5, 17182 doi: 10.1038/srep17182.

[23] Caravan, P.; Farrar, C. T.; Frullano, L.; Uppal, R. Influence of molecular parameters and increasing magnetic field strength on relaxivity of gadolinium- and manganese-based T1 contrast agents. Contrast Media Mol. Imaging 2009, Mar-Apr, 4(2), 89-100.

[24] Varani, James PhD; DaSilva, Marissa BS; Warner, Roscoe L. PhD; Deming, Monica O'Brien; Barron, Adam G. BS; Johnson, Kent J. MD; Swartz, Richard D. MD. Effects of Gadolinium-Based Magnetic Resonance Imaging Contrast Agents on Human Skin in Organ Culture and Human Skin Fibroblasts. Invest. Radiol. 2009, 44(2), 74-81.

[25] Heinrich, Marc C MD; Kuhlmann, Martin K. MD; Kohlbacher, Sonja; Scheer, Mario; Grgic, Aleksandar MD; Heckmann, Martina B.; Uder, Michael MD. Cytotoxicity of Iodinated and Gadolinium-based Contrast Agents in Renal Tubular Cells at Angiographic Concentrations: In Vitro Study. Radiology, 2007, 242(2).

[26] Srinivas, M.; Heerschap, A.; Ahrens, E.T.; Figdor, C.G.; de Vries, I. J. M. ${ }^{19}$ F MRI for quantitative in vivo cell tracking. Trends Biotechnol. 2010, 28, 363-370.

[27] Díaz-López, R.; Tsapis, N.; Fattal, E. Liquid perfluorocarbons as contrast agents for ultrasonography and 19F-MRI. Pharm. Res. 2010, 27 (1), 1-16.

[28] Mizukami, S.; Takikawa , R.; Sugihara, F.; Hori, Y.;Tochio, H.; Wälchli, M.;Shirakawa, 
M.;Kikuchi, K. Paramagnetic Relaxation-Based ${ }^{19}$ F MRI Probe To Detect Protease Activity, J. Am. Chem. Soc. 2008, 130, 794-795.

[29] Xu, S.; Li, D.; Wu, P. One pot facile and versatile synthesis of Monolayer $\mathrm{MoS}_{2} / \mathrm{WS}_{2}$ quantum dots as bioimaging probes and efficient electrocatalysts for hydrogen Evolution Reaction. Adv. Funct. Mater. 2015, 25, 1127-1136.

[30] Mathkar, A.; Narayanan, T.N.; Alemany, L. B.; Cox, P.; Nguyen, P.; Gao, G.; Chang, P.; Aburto, R.R.; Mani, S.A; Ajayan, P. M. Synthesis of Fluorinated graphene oxide and its Amphiphobic properties. Part. Part. Syst. Charact. 2013, 30, 266-272.

[31] Gopalakrishnan, D.; Damien, D.; Shaijumon, M. M. MoS 2 Quantum Dot-Interspersed exfoliated MoS2 nanosheets. ACS Nano 2014, 5297-5303.

[32] Liu, H.; Liu, Y.; Zhu, D. Chemical Doping of Graphene. J. Mater. Chem. 2011, 21, 3335.

[33] Casiraghi, C.; Pisana, S.; Novoselov, K. S.; Geim, A. K.; Ferrari, A. C. Raman Fingerprint of Charged Impurities in Graphene. Appl. Phys. Lett. 2007, 91, 12-14.

[34] Eckmann, A.; Felten, A.; Mishchenko, A.; Britnell, L.; Krupke, R.; Novoselov, K. S.; Casiraghi, C. Probing the Nature of Defects in Graphene by Raman Spectroscopy. Nano Lett. 2012, 12, 3925-3930.

[35] Childres, I.; Jauregui, L.; Park, W.; Cao, H.; Chen, Y. Raman Spectroscopy of Graphene and Related Materials. New Dev. Phot. Mater. Res. 2013, 1-20.

[36] Kim, S.; Hee Shin, D.; Oh Kim, C.; Seok Kang, S.; Sin Joo, S.; Choi, S. H.; Won Hwang, S.; Sone, C. Size-Dependence of Raman Scattering from Graphene Quantum Dots: Interplay between Shape and Thickness. Appl. Phys. Lett. 2013, 102, 2014-2017.

[37] Cancado, L. G.; Pimenta, M. A.; Neves, B. R. A.; Dantas, M. S. S.; Jorio, A. Influence of the Atomic Structure on the Raman Spectra of Graphite Edges. Phys. Rev. Lett. 2004, 93, 
5-8.

[38] Ferrari, A. C.; Basko, D. M. Raman spectroscopy as a versatile tool for studying the properties of graphene. Nat. Nanotechnol. April 2013, 8, 235-246.

[39] Peng, Juan; Gao, Wei; Gupta, Bipin Kumar; Liu, Zheng; Rebeca Romero-Aburto; Ge, Liehui; Song, Li; Lawrence B. Alemany; Zhan, Xiaobo; Gao, Guanhui; Sajna Antony Vithayathil; Kaipparettu, Benny Abraham; Marti, Angel A., Takuya Hayashi, Jun-Jie Zhu, Ajayan, Pulickel M. Graphene Quantum Dots Derived from Carbon Fibers. Nano Lett. 2012, 12, 844-849.

[40] Liu, Fei; Jang, Min-Ho; Ha, Hyun Dong; Kim, Je-Hyung; Cho, Yong-Hoon; Tae Seok Seo. Facile Synthetic Method for Pristine Graphene Quantum Dots and Graphene Oxide Quantum Dots: Origin of Blue and Green Luminescence. Adv. Mater. 2013, 25(27), 36573662.

[41] Dillon, E. P.; Crouse, C. A.; Barron, A. R. Synthesis, characterization, and carbon dioxide adsorption of covalently attached polyethyleneimine-functionalized single-wall carbon nanotubes. ACS Nano 2008, 2, 156-164.

[42] Palchan, I.; Crespin, M.; Estrade-Szwarckopf, H.; Rousseau, B. Graphite Fluorides: An XPS Study of a New Type of CF Bonding. Chem. Phys. Lett.1989, 157, 321-327.

[43] Nansé, G.; Papirer, E.; Fioux, P.; Moguet, F.; Tressaud, A. Fluorination of Carbon Blacks: An X-Ray Photoelectron Spectroscopy Study: I. A Literature Review of XPS Studies of Fluorinated Carbons. XPS Investigation of Some Reference Compounds. Carbon N. Y. 1997, 35, 175-194.

[44] Kresse,G.; Furthmüller, J. Efficiency of ab-initio total energy calculations for metals and semiconductors using a plane-wave basis set. Comput. Mat. Sci. 1996, 6, 15. 
[45] Kresse, G.; Furthmüller, J. Efficient iterative schemes for ab initio total-energy calculations using a plane-wave basis set. Phys. Rev. B 1996, 54, 11169.

[46] Elliott H. Lieb, wo theorems on the Hubbard model, Phys. Rev. Lett. 1989, 62, 1201.

[47] Blochl, P.E. Projector augmented-wave method. Phys. Rev. B 1994, 50, 17953.

[48] Perdew, J. P.; Burke, K.; Ernzerhof, M. Generalized gradient approximation made simple. Phys. Rev. Lett. 1996, 77, 3865.

[49] Monkhorst, J. H.; Pack, J. D. Special packs for Brillouin zone integrations, Phys. Rev. B. 1976, 13, 5188 .

[50] Rohrer, M.; Bauer, H.; Mintorovitch, J.; Requardt, M.; Weinmann, H.-J. Comparison of Magnetic Properties of MRI Contrast Media Solutions at Different Magnetic Field Strengths. Invest. Radiol. 2005, 40, 715-724.

[51] Yang, K. et. al. Multimodal imaging guided Photothermal therapy using Functionalizaed graphene nanosheets Anchored with magnetic nanoparticles. Adv. Mat. 2012, 24, 18681872.

[52] Casolo, S.; Larsen, E.F.; Løvvik, O. M.; Darling, G. R.; Tantardini, G. F. Role of the selfinteraction error in studying chemisorption on graphene from first-principles. Phys. Rev. B 2010, 81, 205412.

[53] Kim, H. J.; Cho, J. H. Fluorine-induced local magnetic moment in graphene: A hybrid DFT study, Phys. Rev. B 2013, 87, 174435.

[54] Perdew, J. P.; Ernzerhof, M.; Burke, K. Rationale for mixing exact exchange with density functional approximations. J. Chem. Phys. 1996,105, 9982.

[55] Adamo, C.; Barone, V. Toward reliable density functional methods without adjustable parameters: The PBE0 model. J. Chem. Phys. 1999, 110, 6158. 
Figure. 1. Schematic illustration of the top down method for the synthesis of fluorinated graphene quantum dots from Fluorinated Graphene Oxide (FGO). FGO is an oxidized derivative of Fluorinated Graphite having $\mathrm{OH}$ and $\mathrm{COOH}$ functional groups on the surface in addition to Fluorine atoms. It is further exfoliated by sonication and stirring at high temperature to break them down into quantum dots.

Figure. 2. a) Bright field Transmission Electron Microscope(TEM) image showing clusters of quantum dots, size distribution with a narrow distribution from 8-12nm, and High resolution TEM image of FGQds the marked area showing the lattice with the SAED pattern as inset confirming the crystalline nature b) Raman spectra of FGQds showing the characteristic G peaks associated with graphene and the defect peaks D, 2D and D+D' arising from the introduction of defects due to fluorination and functionalization c) FTIR spectrum of FGQds the peaks corresponding to the functionalities are marked. The inset shows the entire spectrum showing the presence of $\mathrm{C}-\mathrm{F}$ bond.

Figure. 3. Deconvoluted XPS spectrum a) C1s spectra of FGQds showing sp2 C and C-F bonds b) F1s spectra of FGQds with a single peak corresponding to the C-F bonding c) SQUID hysteresis curve for FGQds, the linear dependence of the Magnetization on the applied field showing paramagnetic behavior is seen d) ZFC curve showing the experimental and fitted curves. The inset shows the curie's law dependence showing the linear dependence of inverse of susceptibility on temperature confirming the paramagnetism.

Figure. 4. a) Energy difference $(\Delta \mathrm{E})$ between total energy of magnetic and nonmagnetic solution of the functionalized graphene b) Total magnetic moment of the functionalized graphene. c) d) and e) Spin charge density plot of $\mathrm{F}, \mathrm{OH}$ and, $\mathrm{COOH}$ functionalized graphene respectively. The 
spin up and spin down charge density is represented by blue and yellow color respectively. The $\mathrm{C}, \mathrm{F}, \mathrm{O}$ and $\mathrm{H}$ atoms is depicted by cyan, red, magenta and blue color balls, respectively.

Figure. 5. a) The image of a slice acquired during the proton MRI showing the contrast where the paramagnetic FGQds are present b) Image acquired during 19F MRI showing the relaxation of $19 \mathrm{~F}$ nuclei giving a contrast c) schematic showing the mechanism of relaxation of protons in water by the quantum dots. When the RF pulse is applied the local magnetic field created by the quantum dots interact with the protons in water shortening of the $T_{2}$ relaxation time $\left.d\right)$ The results of MTS assay of the HEK 293T cells incubated with the quantum dots at different concentrations for 1, 2 and 3 days e) Images of GFP labeled SUM 159 cells incubated with the highest concentration of quantum dots after 1-day f) After 3days of incubation, inset shows an overlapped bright field and fluorescent image. 


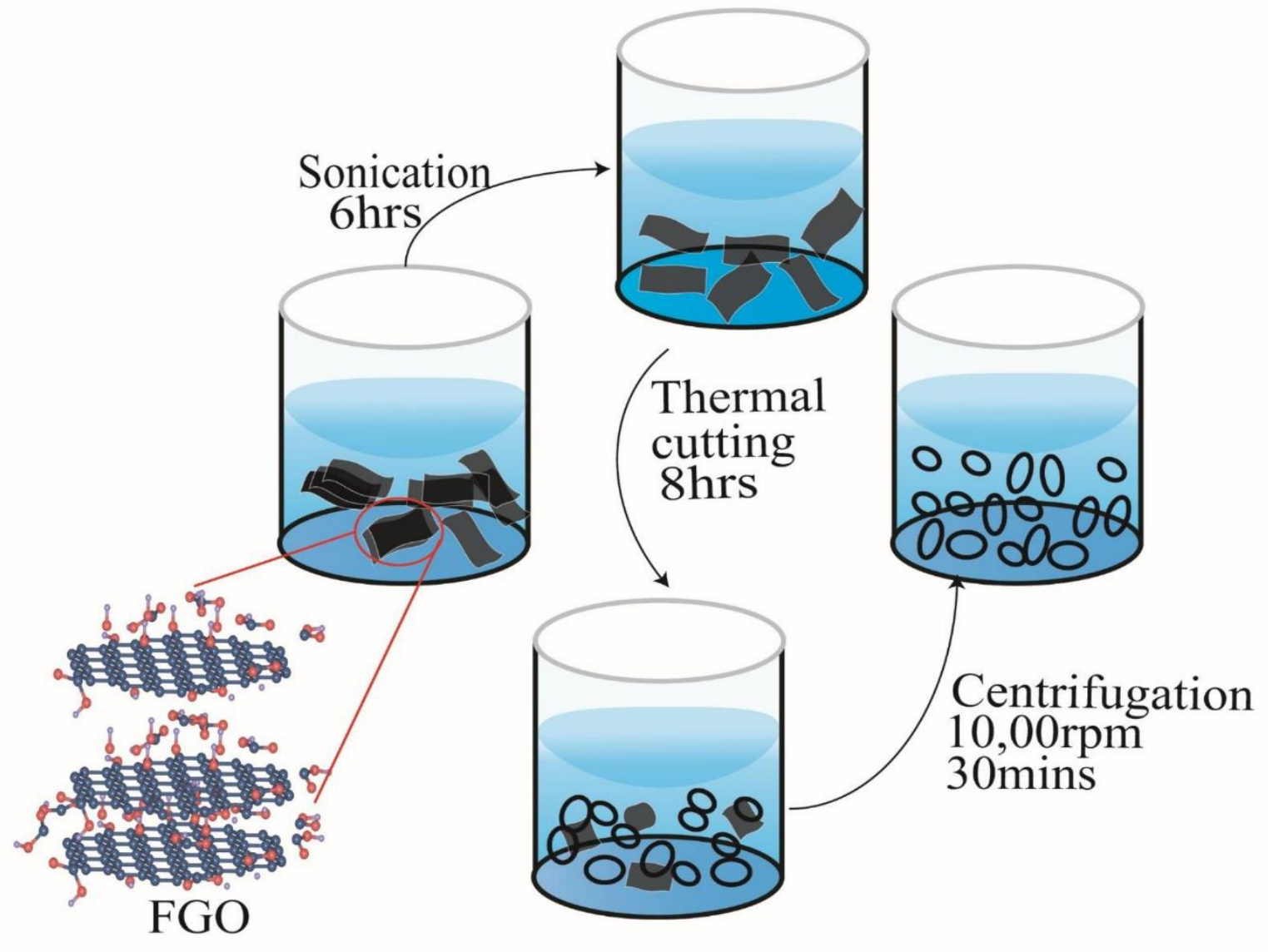

Figure.1 
a)
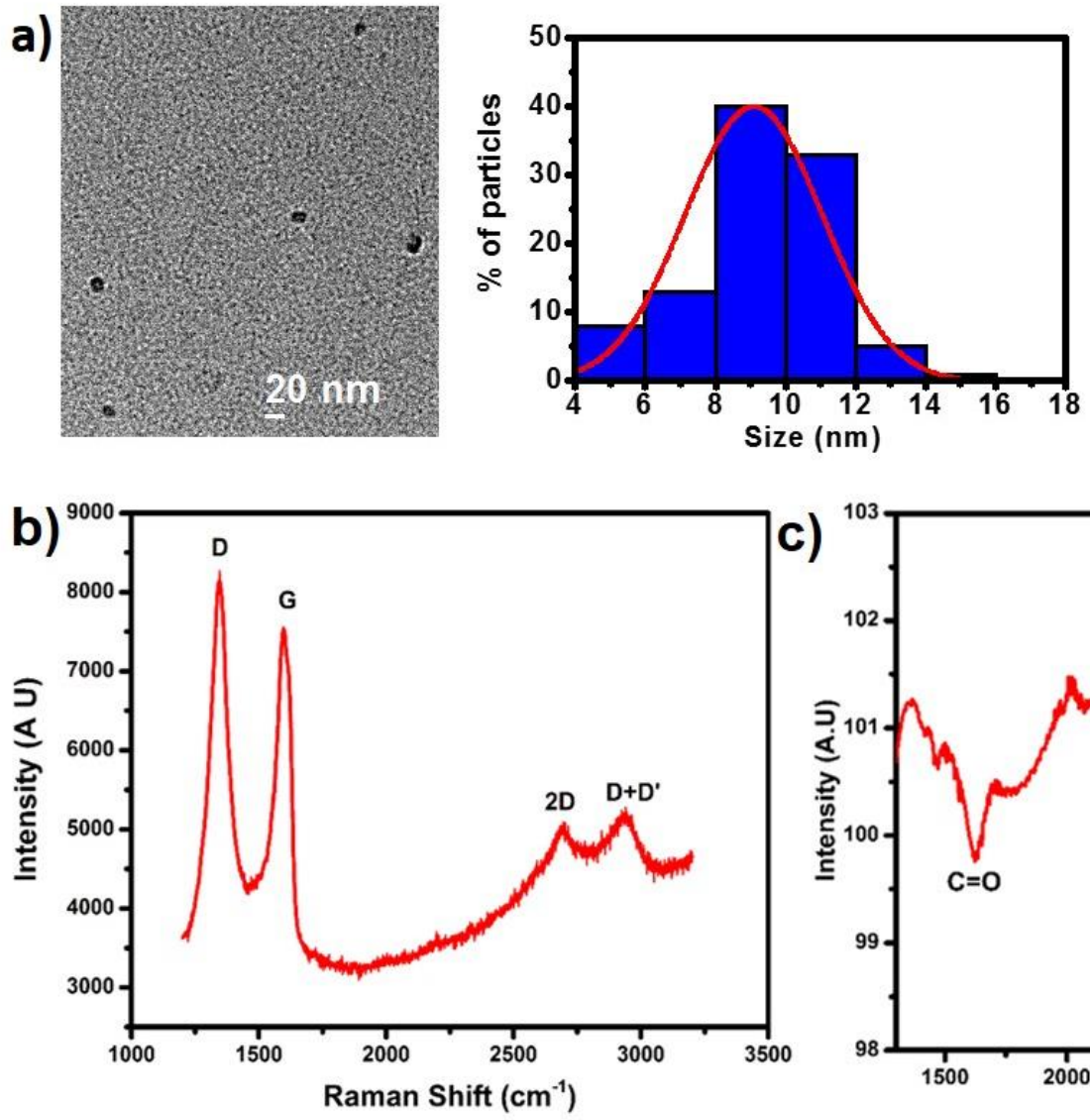
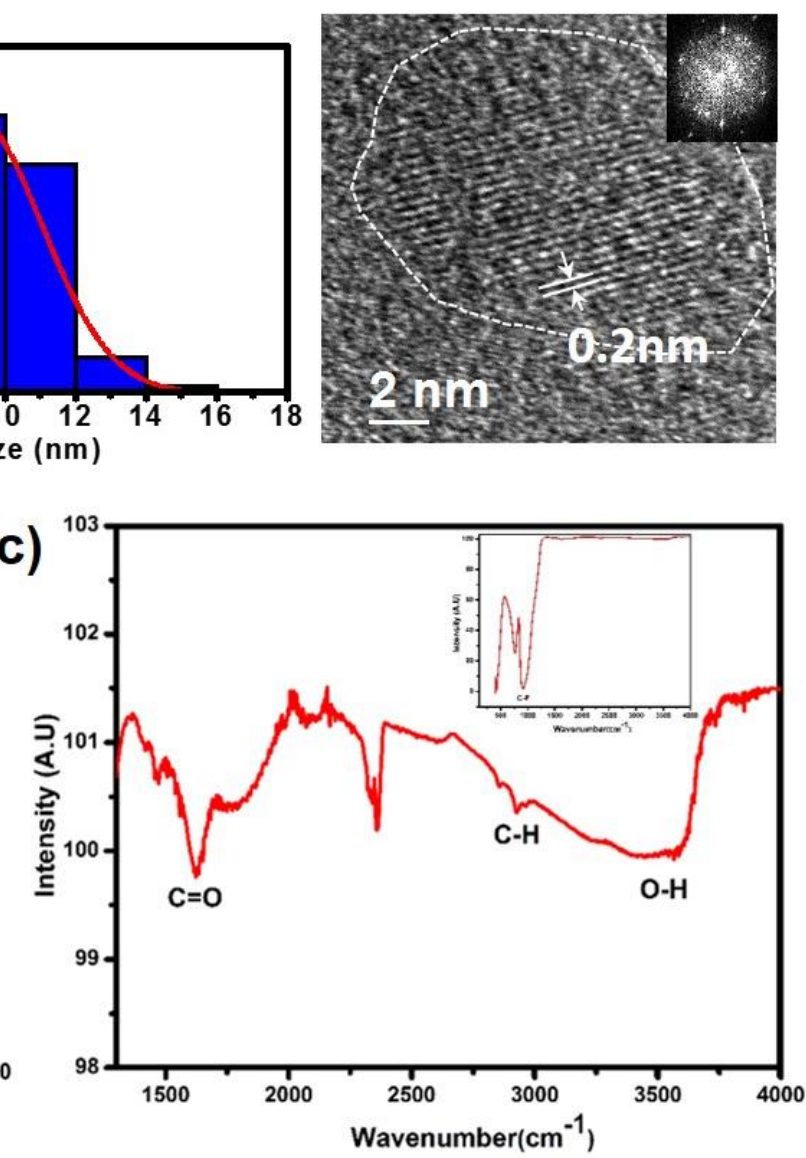

Figure.2 

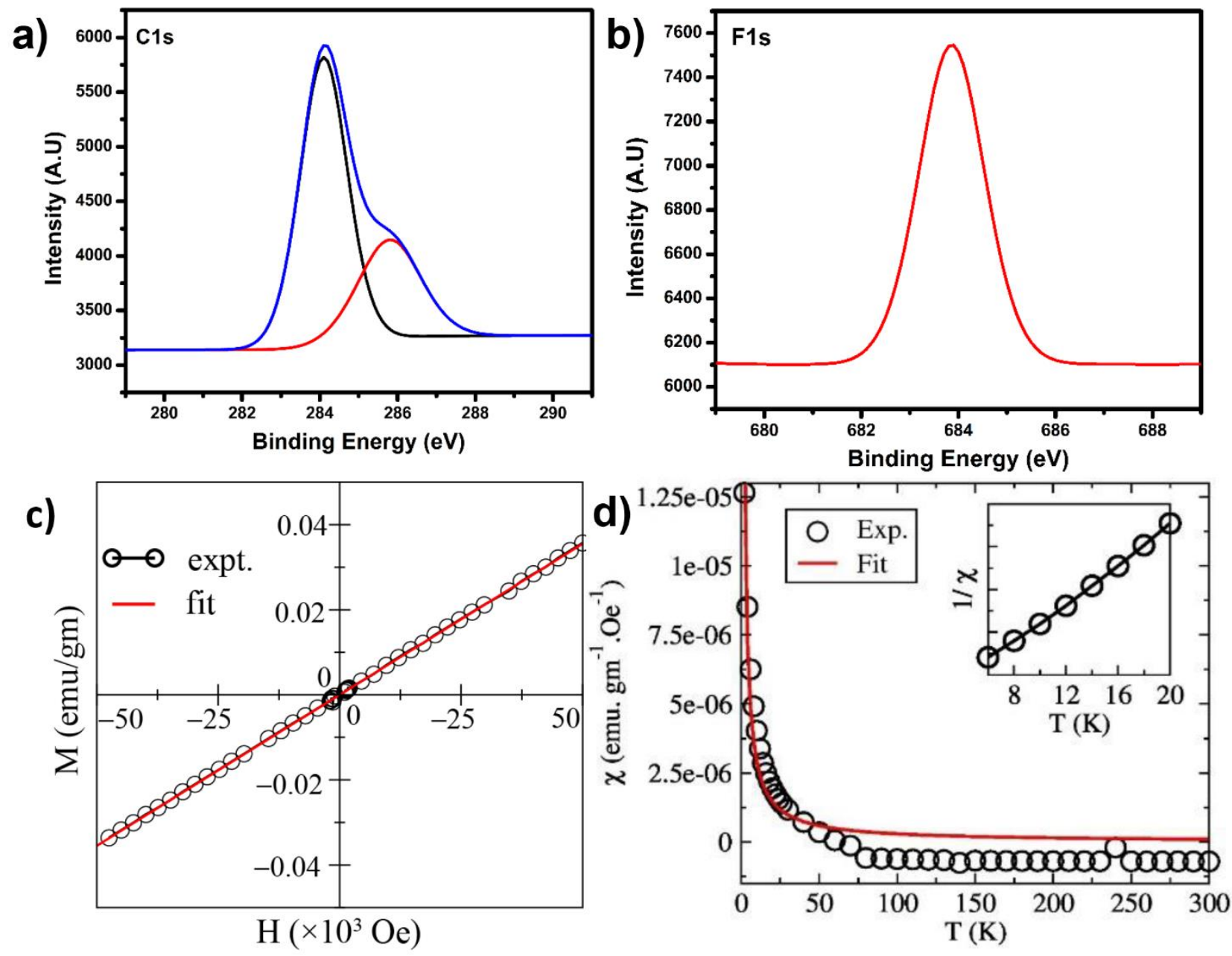

Figure.3 

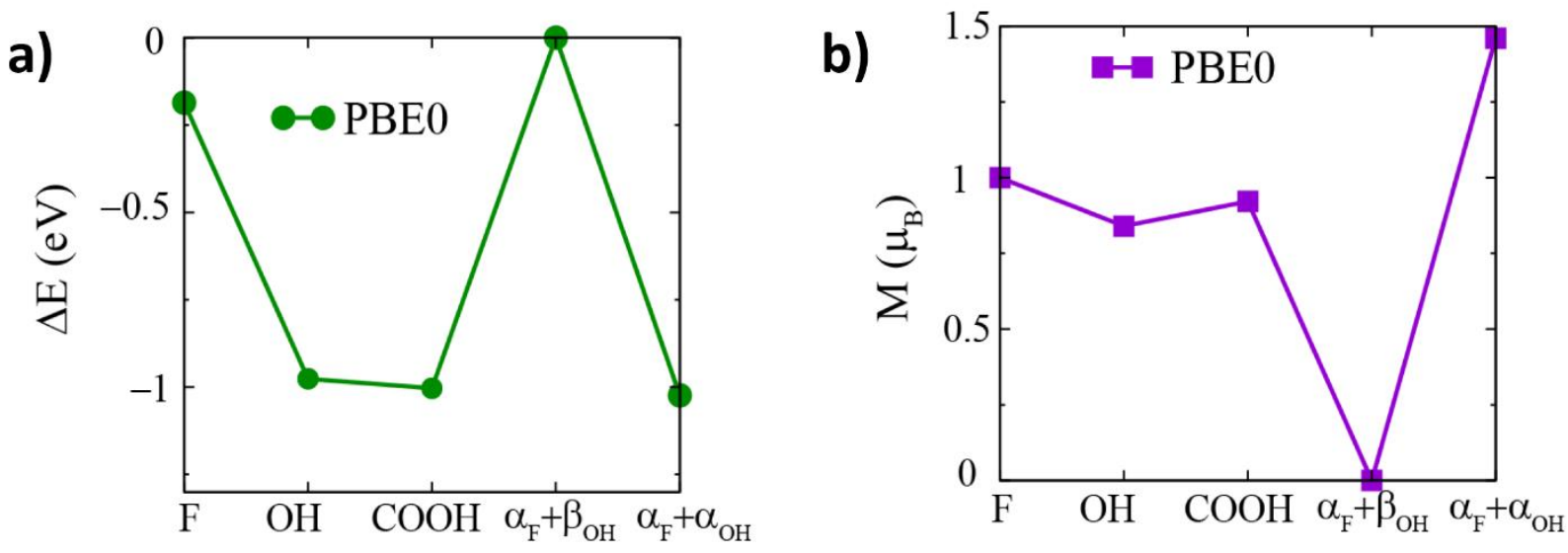

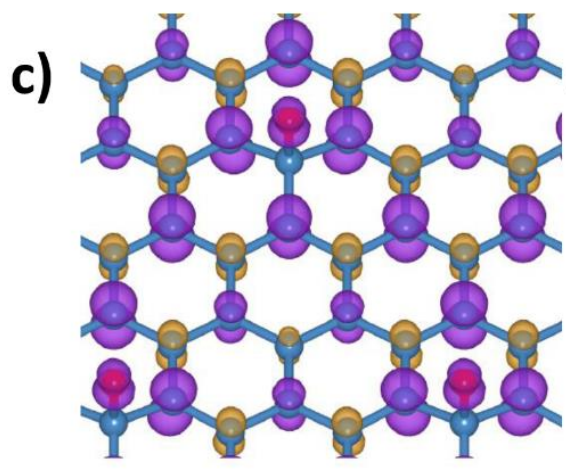

GaF

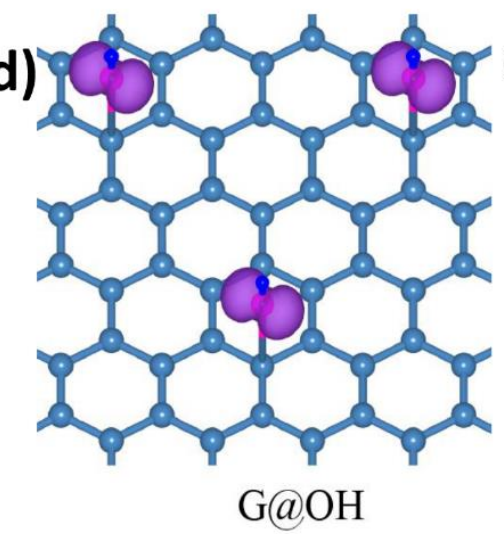

Figure.4

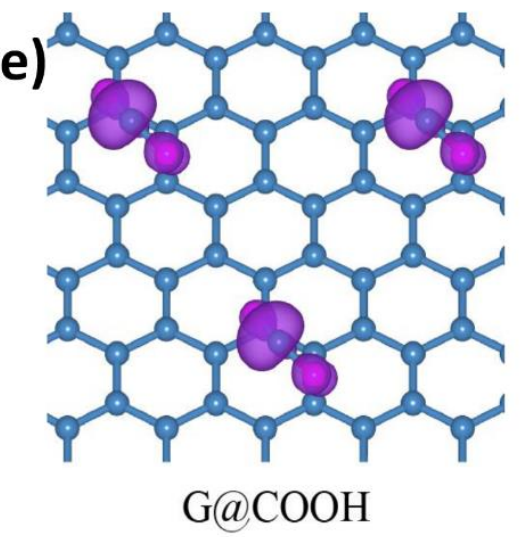

$\mathrm{G} @ \mathrm{COOH}$ 

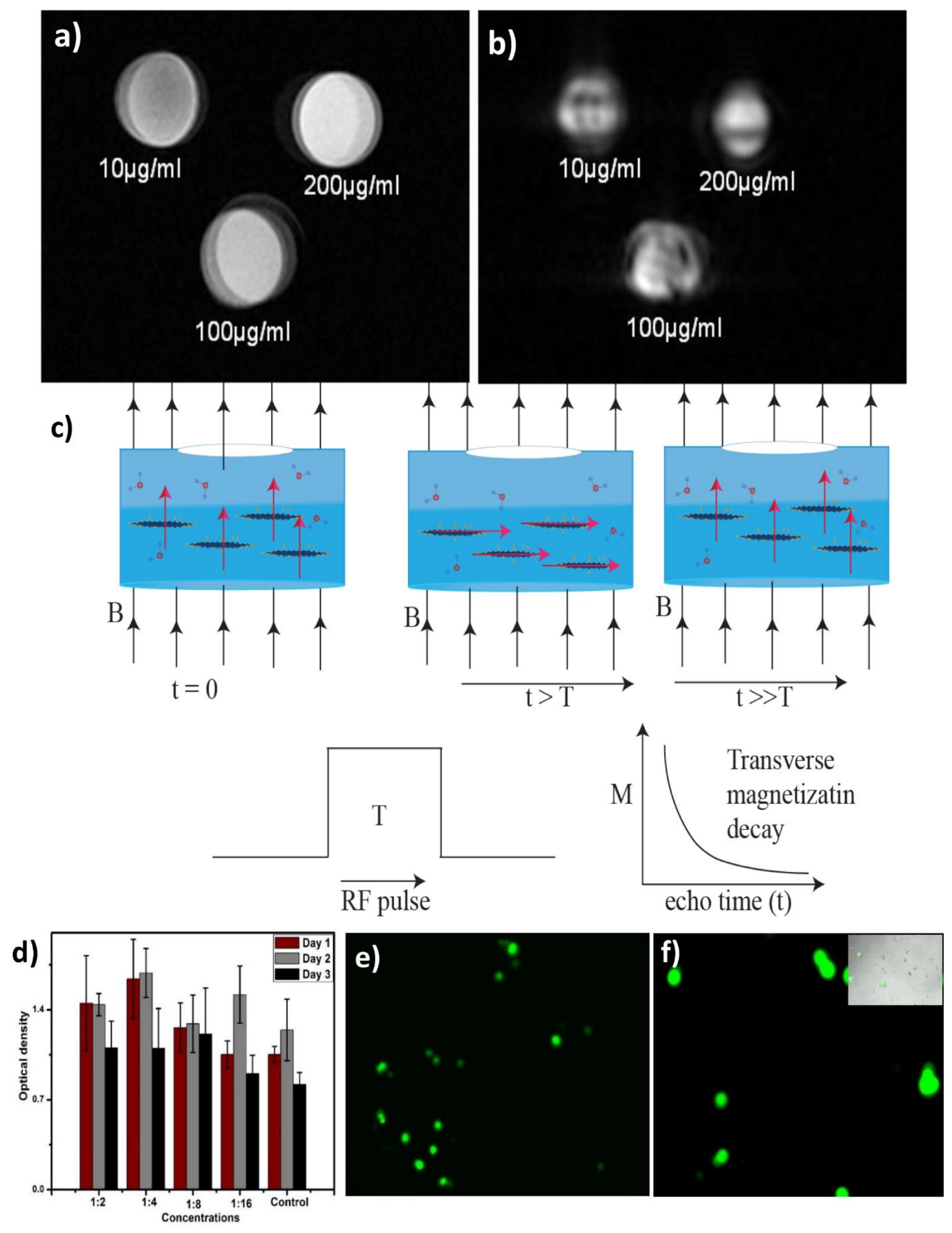

Figure.5 


\section{The table of contents entry:}

Keywords: Fluorinated graphene Quantum dots, Magnetic Resonance Imaging (MRI), Graphene, Paramagnetism, Contrast agent

Sruthi Radhakrishnan ${ }^{1}$, Atanu Samanta ${ }^{2}$, Parambath M. Sudeep ${ }^{1}$, Kiersten L. Maldonado ${ }^{3}$, Sendurai A. Mani ${ }^{4}$, Ghanashyam S. Acharya ${ }^{5}$, Chandrasekhar S. Tiwary ${ }^{1, *}$, Abhishek K. Singh ${ }^{2, *}$, Pulickel M. Ajayan ${ }^{1, *}$

\section{Metal Free Dual Modal Contrast Agents based on Fluorographene Quantum Dots}

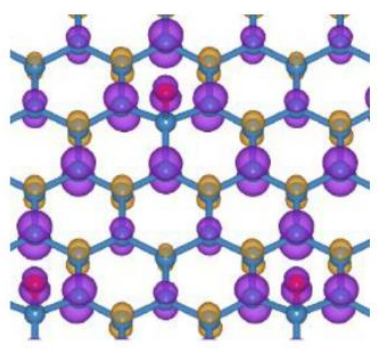

G@F

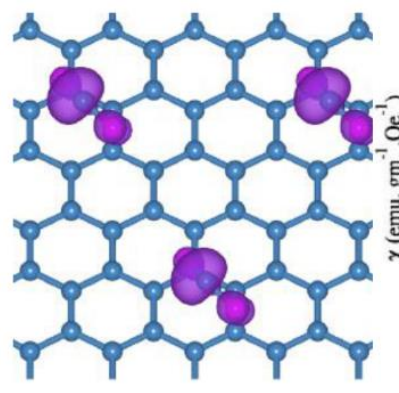

$\mathrm{G}(\mathrm{COOH}$

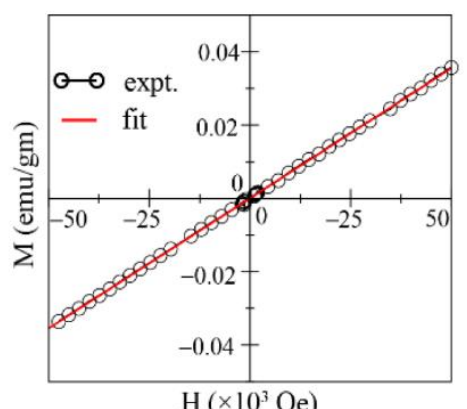

$\mathrm{H}\left(\times 10^{3} \mathrm{Oe}\right)$

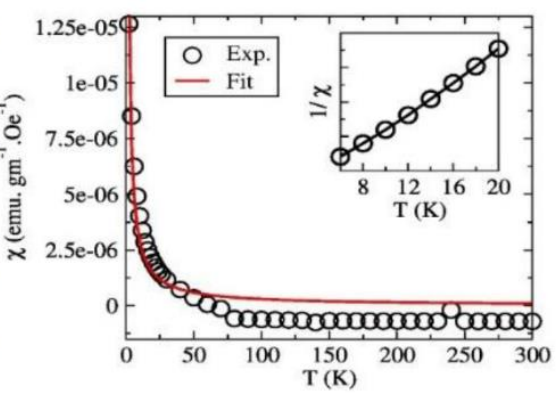

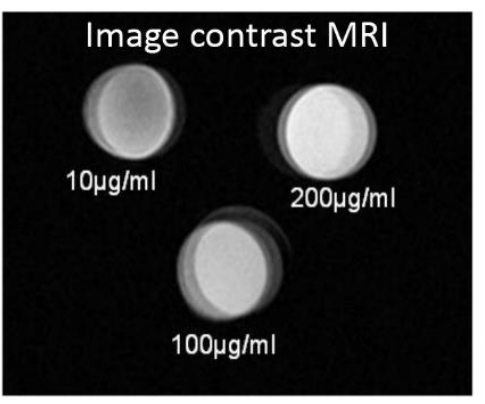

Image contrast in 19F MRI

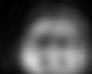

$10 \mu \mathrm{g} / \mathrm{ml}$

$200 \mu \mathrm{g} / \mathrm{ml}$

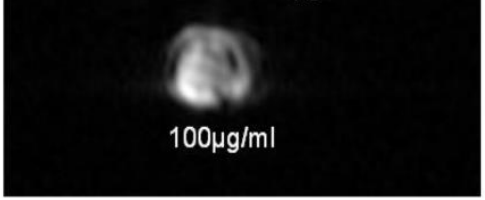

TOC: Schematic shows a representative of the systems of fluoro-graphene quantum dots studied here. Non-functionalized and functionalized fluoro-graphene systems are studied of which acid functionalized system shows greater magnetic moment. This is experimentally verified and the

hysteresis and low temperature studies reveal paramagnetic behaviour. The inherent paramagnetism along with the fluorine content is utilized in its development as a dual modal agent for proton and $19 \mathrm{~F} \mathrm{MRI}$. 